\title{
El trabajo de campo \\ como colaboración en la diáspora: Iglesia, ONGs y trabajadoras domésticas filipinas en Roma
}

\author{
MARGARET MAGAT \\ University of Pennsylvania. Filadelfia. USA
}

\section{RESUMEN}

$\mathrm{Al}$ realizar trabajo de campo, una de las cuestiones clave es el tema de la colaboración con distintos individuos o grupos. Este artículo utiliza las "narrativas de la colaboración" como un medio para analizar las relaciones establecidas con sacerdotes, trabajadores de organizaciones no gubernamentales y oficiales de la Embajada filipina, tratando, al mismo tiempo, de mantener la confianza de las colaboradoras principales: las trabajadoras domésticas filipinas. El análisis incluye una exploración de los problemas que se derivan de las relaciones establecidas, así como el papel simultáneo de la etnógrafa desde fuera y desde dentro de la comunidad, y pretende contribuir a entender cómo la comunidad migrante filipina en Roma construye el conocimiento y consigue credibilidad y legitimación.

Palabras clave: Métodos antropológicos, Colaboración, Filipinos, Migrantes, Trabajadores domésticos.

\section{SUMMARY}

In conducting ethnographic research, collaboration with various individuals and groups remain critical. Through "narratives of collaboration," this article discusses the establishment of partnerships from priests to non-government organization workers (NGOs) as well as Philippine embassy officials, while maintaining the trust of the main collaborators, the Filipina domestic workers themselves. It explores the problems stemming from collaborations and from the ethnographer's insider/outsider status in the community. This work looks at the construction of knowledge in the Filipino migrant community in Rome and how it gains credibility and legitimacy.

Key words: Anthropological Methods, Collaboration, Filipino, Migrants, Domestic Workers.

RDTP, LVIII, 1 (2003): 171-190 
Una negra noche de mitad de diciembre aterricé en Roma con dos sensaciones contradictorias: me sentía feliz y, al mismo tiempo, preocupada. Feliz porque iba a empezar mi trabajo de campo entre las inmigrantes filipinas que trabajan como empleadas domésticas y también, preocupada porque no conocía personalmente a nadie en la Ciudad Eterna, mis únicos contactos consistían en nombres y números escritos en papel. Y justo en esa situación me di cuenta de que había perdido mi monedero y que el tren que me tenía que llevar al centro de la ciudad estaba a punto de salir. Encontré el monedero en la estación de policía, corrí para coger el tren y una pareja filipina me ayudó a subir el equipaje. Esas dos personas acabarían convirtiéndose en los primeros colaboradores de una larga lista de personas con las que tuve la suerte de trabajar durante mi estancia en Roma.

Aprendí en seguida que para entender el modo de vida, siempre cambiante, de los filipinos en Roma, era no sólo deseable, sino imprescindible, trabajar en colaboración con ellos. Se estima que unos 200.000 filipinos viven en Italia contratados como empleados domésticos, pero la mayor parte de este total está constituido por mujeres. En otras partes del mundo, tales como Arabia Saudita, Grecia, Singapur o Hong Kong, el hecho de que mujeres filipinas con estudios trabajen como empleadas del hogar está tan extendido que el apelativo con el que se nombra a este grupo se ha convertido en un sinónimo de su trabajo (Chell-Robinson 2000; Iosifides 1997; Lazaridis 2000; Constable 1997). Por ejemplo en la propia Italia, el término "Filippina" significa "chica de servicio" y en Hong Kong "banmui", que se traduce como "chica filipina", significa también "sirvienta" o "empleada de hogar".

El presente trabajo está basado en la investigación que he realizado para mi tesis doctoral, dedicada a analizar si la migración laboral conduce a un asentamiento cultural en la diáspora filipina. Mi objetivo es explorar la forma en la que las mujeres establecen su localidad y su etnicidad a partir de su sitio, de su cultura material o de otro tipo de expresiones culturales, centrando el estudio en la vida y experiencias de las trabajadoras domésticas en Roma. Voy a dedicar este artículo a discutir cómo he establecido las relaciones con mis colaboradores, deteniéndome en los problemas y en el rol que ellos me asignaron a mí, en un lugar dentro/ fuera de la comunidad (Narayan 1989). Me interesa también prestar atención a la construcción del conocimiento en el grupo y a la credibilidad y legitimación con la que éste se presenta, para ello voy a emplear algunas de las "narrativas de la colaboración" en las que se subraya la necesidad de trabajar con informantes que cubran un amplio espectro, incluyendo: sacerdotes, trabajadores de ONGs, empleados de la Embajada Filipina, pero 
manteniendo la confianza depositada en mí por mis colaboradores de mayor importancia: las mujeres migrantes.

Yo misma soy una filipina-americana, nacida y criada en Filipinas, pero residente en Estados Unidos desde hace dieciocho años, a lo largo de los cuales adopté también la nacionalidad de este país. Por este motivo soy consciente de mi doble estatus de dentro/fuera de la comunidad entre la que he realizado el trabajo de campo. Me considero parte del grupo, debido a mi adscripción étnica, pero a pesar de ello eran patentes algunas diferencias importantes, tales como la clase, la generación, la educación e incluso la cultura (véase Vo 2000: 19), y aunque hablo con fluidez el tagalo (la lengua principal en Filipinas) e incluso me he sentido aceptada por la mayor parte de los filipinos en Roma, en algunas ocasiones me he visto desempeñando el incómodo papel del "Otro". Creo que mi sentimiento de no pertenecer, de estar fuera, de ser un observador externo, va en cierto modo limitado por la propia posición exterior de la mayoría de los filipinos que he conocido en Italia; así que, de alguna forma, yo estaba tomando contacto con personas que habitaban y trabajaban en un terreno liminar, y que coincidía con la posición que yo misma ocupaba como etnógrafa.

Realicé el trabajo de campo entre noviembre del año 2000 y junio del 2001, pero volví brevemente en febrero del 2002 para encontrarme con algunos de mis colaboradores y saber qué tal les estaba yendo. La metodología empleada ha estado constituida por la observación participante y la realización de entrevistas en profundidad, cuya duración cubre un espectro que va desde las dos horas a varios días. La mayor parte de mi información procede, en concreto, de catorce mujeres filipinas a quienes he acompañado en sus salidas y en sus recados durante el trabajo de campo, con cuya generosidad me siento en deuda, y con quienes, en su mayor parte, me mantengo en contacto por correspondencia. Entrevisté, además, a una docena de oficiales y líderes comunitarios, tanto hombres como mujeres, e incrementé mis fuentes de información siempre que pude hablando con filipinos que encontré en las calles de Roma. Me dirigía a las mujeres empleando el tagalo porque soy consciente de la existencia de "las habilidades de comunicación y los repertorios nativos que usa la comunidad" (Briggs 1986: xi), sin embargo mi fluidez en esta lengua ha quedado reducida por el hecho de que he vivido los últimos diez años en los Estados Unidos, algunas veces me han tenido que explicar determinados acontecimientos ocurridos en Filipinas y yo misma me he convertido a veces en la diana del discurso anti-americano. Esta última faceta de mi papel tiene que ver con la imagen de los Estados Unidos como la tierra del maná y el capitalismo que provocaba quejas, especialmente entre los trabajadores 
de las ONGs, mayoritariamente de izquierdas, acerca de la explotación a la que están sometidos los empleados domésticos en particular y las mujeres en general, y su frustración ante la economía globalizada. Esto provocó en mí una sensación de desilusión que arrastré durante el trabajo de campo, pero no discutía con ellos, puesto que mi objetivo era saber por qué la gente hace lo que hace (véase Vo 2000: 23). El hecho de que yo misma fuera filipina, alguien que compartía su etnicidad, provocó una serie de problemas particularmente complejos como consecuencia de mi identidad, tanto profesional como personal (Kang 2000: 39).

Desde que me instalé en una habitación que alquilé a una mujer italiana en el distrito romano de Piramide, empecé a encontrarme con mujeres filipinas en paradas de autobús, plazas, iglesias o simplemente paseando. Me di cuenta en seguida de que en cada zona de Roma existía una plaza o terminal de transporte donde se reunían los filipinos y allí socializaban, comían, jugaban y pasaban su tiempo libre. Casi siempre les resultaba curioso verme vestida con vaqueros y mochila a la espalda donde guardaba el cassette, bolígrafos, un cuaderno y una ubicua botella de agua; pero siempre fui bienvenida en esa comunidad estrecha, en esa red de amistades, quizá precisamente porque sabían que no trabajaba en Roma como empleada del hogar. La confianza, una parte integral del trabajo de campo, resultó ser crítica desde el primer momento en que conocía a una mujer; el hecho de que yo no fuera vista como alguien que les podía quitar el trabajo, indudablemente contribuyó a construir unas relaciones de confianza. Cuando me presentaba como una estudiante de los Estados Unidos interesada en las migrantes filipinas, mis interlocutoras empezaban por mirarme de arriba abajo, haciendo algunas veces comentarios sobre mis zapatos que eran siempre cómodos, negros y adecuados para caminar, a diferencia de las zapatillas de deporte que suelen llevar los turistas americanos. Los zapatos fueron mi única concesión a la moda italiana, en el sentido de que no quería parecer una auténtica turista. Algunas mujeres me llegaron a decir con el tiempo que dedujeron que yo no trabajaba como empleada doméstica precisamente debido al tipo de zapatos que llevaba.

Una de las quejas más frecuentes que escuché fue la de que, tanto hombres como mujeres, en alguna ocasión han ayudado a un compatriota ofreciéndoles actuar como substitutos en sus trabajos mientras hacían una visita a Filipinas o durante las vacaciones, para encontrarse, al volver, con el hecho de que habían sido reemplazados permanentemente por ellos, ya que habían ofrecido sus servicios por un precio más bajo. Este tipo de narrativas y otras relacionadas con el desengaño causado por pseudo-amigos influyeron en el contexto de mis primeros encuentros con 
las mujeres; sin embargo, el tiempo, la participación en los acontecimientos organizados por la comunidad y el hecho de que yo era vista frecuentemente en compañía del sacerdote más importante del grupo, el Padre Bati, y otros sacerdotes, allanaron el camino de mi aceptación. Cuando esto ocurrió, las primeras mujeres empezaron a referirme a sus amigas y conocidas y así conseguí los nombres de nuevas personas para entrevistar, empleando lo que se conoce como el "método de la bola de nieve".

Algunas mujeres hicieron explícita su sorpresa, y a veces incluso su orgullo, al conocer a otra filipina que no trabajaba como empleada del hogar. Al mismo tiempo, y conocida mi nacionalidad estadounidense, fui objeto, con frecuencia, de demandas emocionales de ayuda para conseguir trabajo en los Estados Unidos o un marido. En muy pocos casos fui vista como una persona rica y privilegiada de la que se podía obtener ventaja como ciudadana norteamericana, pero la mayoría de las veces, el hecho de ser estudiante, filipina-americana, católica y mujer me permitió ejercer una "flexibilidad de situación" en el campo (Vo 2000: 22).

Casi nunca fue problema contactar con mujeres filipinas, pero llegar a compartir con ellas un tiempo de calidad sí que lo fue. Sus únicos días libres eran los jueves por la tarde y los domingos, pero algunas mujeres trabajaban incluso esas horas para enviar más dinero a sus casas. Lo que solía hacer era acompañarlas durante los periodos entre los trabajos, a la iglesia, o en los recados que tenían que hacer para la casa donde trabajaban. La mayoría de las veces me encontraba con ellas fuera de sus ambientes de trabajo y de residencia, porque era como lo preferían, y también porque mi propio objetivo se centraba en las experiencias culturales de las mujeres fuera de la casa.

Además de la dificultad de programar bloques de tiempo para realizar las entrevistas, yo mantenía citas con personas, tanto italianas como filipinas, que desempeñaban puestos oficiales, y relacionarse a la vez en Roma con organizaciones y con individuos constituye una actividad delicada. Las personas que trabajan para la embajada filipina son vistas, generalmente, como gente que se aprovecha de los trabajadores migrantes, y esta percepción coincide, a su vez, con la experiencia y la observación de los miembros de las ONGs. Sin embargo, la relación estrecha que existe entre la iglesia católica y los trabajadores no religiosos de las ONGs, algunos de los cuales pertenecen al Partido Comunista de Filipinas, ha creado una estructura de sustento para los filipinos, tanto material como espiritual, a la que me voy a referir más adelante.

Filipinas, un país constituido por más de 7.000 islas, es el único estado católico-romano en el sureste asiático. Descubierto por Magallanes en 1521, fue denominado así en honor a Felipe II de España y fue una colonia es- 
pañola durante trescientos años. En 1896 un grupo llamado Katipunan comenzó un movimiento de protesta en contra de España, pero la rebelión fue aplastada y sus líderes ejecutados (incluido el héroe nacional José Rizal), lo que no impidió que continuara habiendo insurrecciones populares por todo el archipiélago, hasta que en diciembre de 1897 se firmó un pacto que puso fin, temporalmente, a las hostilidades. El 1 de mayo de 1898, durante la guerra hispano-norteamericana, los Estados Unidos atacaron la flota estacionada en la bahía de Manila. La victoria de los americanos en la guerra les configuró, por primera vez, como una potencia, y se aliaron con los rebeldes filipinos en contra del enemigo común, España. El 12 de junio de 1898 el general Aguinaldo declaró la independencia de Filipinas, pero no fue reconocida de forma oficial por los Estados Unidos, enseguida quedó claro que este país tenía sus propios planes para los filipinos:

En agosto los españoles consintieron en luchar una batalla fingida contra las tropas norteamericanas para salvar el honor y evitar que las fuerzas filipinas entraran en la capital. Los revolucionarios filipinos, que no podían reclamar la victoria, tuvieron que establecer su capital en el norte, y la nueva república independiente, incapaz de hacer reconocer su soberanía, acabó, forzosamente, enfrentándose en una nueva contienda contra los nuevos colonos en febrero de 1899 (Rafael 2000: 10).

La sangrienta guerra filipino-norteamericana (1899-1913), vista por algunos como "el primer Vietnam americano" se cobró las vidas de 250.000 hombres, mujeres y niños filipinos, y de 500 americanos. Los Estados Unidos declararon su victoria, de manera oficial, en 1902 y proclamaron que su política colonial en Filipinas iba a ser la de una "asimilación benevolente", pero en realidad "la mentalidad de 'cazadores de indios' explica el comportamiento salvaje de las tropas americanas" (Anderson 1998: 201)1. En 1898 Estados Unidos compró Filipinas y otros territorios (incluido Puerto Rico) a España por 20 millones de dólares y, después de medio siglo como colonia americana, Filipinas consiguió su independencia en 1946. A partir de entonces el país ha luchado por conseguir lo que algunos estudiosos han llamado "desarrollo desigual", legado por el colonialismo (Eviota 1992; Alegado 1992).

Desde 1974, cuando Marcos puso en vigor el Programa de empleo en ultramar para tratar de solucionar el problema del desempleo y conseguir, al mismo tiempo, remesas de dinero extranjero² (Carino y otros 1998;

\footnotetext{
${ }^{1}$ Sobre el tema de la revolución filipina, véase Ileto (1979).

${ }^{2}$ Comunicaciones personales, por teléfono el 25 de febrero, y entrevista personal el 19 de marzo de 1999, con el agregado de trabajo de la Embajada de Filipinas en Washington, D.C.
} 
Parrenas 1998), los trabajadores han ocupado el primer lugar en las exportaciones del país. De un total de 72 millones de habitantes, se estima que unos 4 millones de trabajadores filipinos se encuentran repartidos por 130 países, a los que hay que sumar millones de indocumentados ${ }^{3}$ (Go 1998; Alegado 1992). Y a pesar de que la exportación de "energía humana" fue vista como una solución temporal para estimular la economía, se ha convertido actualmente en una estrategia económica indispensable. Las remesas de dinero enviadas por los migrantes ascendieron a unos siete mil millones de dólares en 1998 (Vanzi 1998). Existen siete bancos filipinos en Roma que envían estas remesas, el gerente de uno de ellos estimaba que el suyo remitía a Filipinas al menos un millón de dólares por semana.

Hace veinte años que Europa está experimentando lo que se denomina "nueva migración", protagonizada por países de África, Asia y Europa del este que han empezado a llegar a lugares tradicionales de emigración, tales como Italia y España, transformándolos en lugares de inmigración (Koser y Lutz 1998; Cole 1997; Anthias y Lazardis 2000). Uno de los principales flujos de este nuevo movimiento está constituido por mujeres, solteras y casadas, que migran para trabajar en el sector doméstico, pero este tema sólo ha empezado a atraer el interés del mundo académico en los últimos años, y la escasa producción al respecto afecta, tanto a los lugares de origen, como a los de destino (véase Koser y Lutz 1998; Phizacklea 1998; King y otros 2000; Anthias y Lazardis 2000; Campani 2000). En Italia la demanda de trabajo doméstico continúa siendo alta, en parte debido al alto número de mujeres que trabaja fuera del hogar y el escaso número de instituciones dedicadas al cuidado de niños y ancianos. Las mujeres filipinas, junto con las caboverdianas, empezaron a llegar a finales de la década de los 60 y durante los años 70 del siglo XX (Andall 1998 y 1999), y a pesar de la presencia de otros colectivos de trabajadoras domésticas (somalíes y latinoamericanas), la filipinas constituyen la mayoría de las empleadas domésticas no sólo en Italia, sino en otros lugares del mundo (Alabastro 1995; Cohen y Vertovec 1999; Parrenas 1998).

Los filipinos que yo he entrevistado en Roma se refieren de forma consensuada a la ciudad como el "Hong Kong de Europa". Roma ejerce en ellos una especial atracción debida, en parte, al hecho de que aloja el Vaticano, la capital de la fe católica. Además es la capital de Italia, un país conocido por la precariedad de sus fronteras, tanto al entrar como al salir. Italia ha experimentado una enorme explosión en el sector informal de la economía, en el que existen nichos laborales específicos que demandan mano de obra barata. Todo ello unido a otro tipo de condiciones como

\footnotetext{
3 Véase nota anterior.
} 
una legislación inadecuada se combina con la cultura de la migración en Filipinas para concebir Italia como un lugar preferente de destino para aquellos filipinos que buscan su propia versión de "la dolce vita". Pero a pesar de estas esperanzas, tanto los filipinos como los migrantes de otros países, al llegar a Italia acaban viviendo, ellos y sus familias, en una situación precaria y difícil, caracterizada por la falta de recursos: el acceso a la vivienda difícil, la violencia doméstica corriente, los embarazos no deseados y la inexistencia de lugares para pasar el tiempo libre, son los aspectos más citados en mis entrevistas. Estos problemas se agravan por el hecho de que las personas que trabajan en la embajada filipina mantienen unas relaciones muy difíciles con los miembros de las ONGs, de manera que los representantes oficiales del país de origen de los trabajadores miran con sospecha cualquier reclamación de las personas a quienes representan. La Embajada dificulta constantemente cualquier trámite burocrático, elevando los precios de los impuestos para realizarlos y de esta forma se enfrentan a las protestas de muchos filipinos que están convencidos de que son sus trabajos y las remesas de dinero que ellos envían a Filipinas lo que mantiene la economía del país a flote.

El trabajo de campo realizado indica que la comunidad filipina ha buscado otras fuentes de información en las que no ha visto traicionada la confianza depositada en ellas a lo largo del tiempo, en su caso concreto se trata de la iglesia católica. Los filipinos tienen en Roma una parroquia oficial, Santa Pudenziana que les ha sido asignada por el Papa Juan Pablo II. El párroco de este centro, dedicado a ejercer "el ministerio de evangelización y servicio pastoral" (Cuchapin 2000: 222), es el Padre Remo Bati. El Padre Bati se encarga de todo en su parroquia, desde oficiar misa y ejercer de consejero matrimonial hasta actuar de intérprete cultural y legal para las autoridades italianas. En una ocasión compartió conmigo una narrativa que ilustraba sus múltiples roles.

Un día recibió una llamada de una mujer filipina que se encontraba retenida en el aeropuerto de Fiumicino sin recursos, las autoridades de inmigración la tenían detenida desde hacía varios días sin acceso a ropa o comida, de manera que el Padre Bati llamó a una de las monjas de la parroquia y le dijo: "hermana, por favor, compre ropa interior de mujer", "¿qué clase de ropa interior?" preguntó la hermana, "un sujetador y unas bragas" contestó el sacerdote con seguridad, "¿de qué talla?" preguntó ella, "no tengo ni idea", dijo él, "jal fin y al cabo soy un sacerdote! ¡simplemente cómprelos!", y tratando de sobreponerse a su turbación, entregó la ropa interior y la comida a la mujer, que quedó libre bajo su protección.

Uno de los problemas que surgió durante mi trabajo fue el de averiguar la fecha y el horario de todo tipo de eventos, incluidas las repre- 
sentaciones culturales, que tenían lugar en la comunidad. Los sacerdotes como el Padre Bati solían saber que determinado acto iba a tener lugar, pero no conocían más detalles, de manera que sólo conseguía enterarme con precisión después de varias llamadas a mis contactos en las ONGs. Durante un evento cultural concreto que tuvo lugar en Santa Pudenziana, y cuya información precisa tuve que obtener a base de varias llamadas telefónicas, me encontré con una de las personas que trabajaba en la embajada, una mujer que no demostró ninguna alegría al verme: "Ah, estás tú aquí", dijo con sorpresa mezclada con desdén, "¿cómo te enteraste?", me preguntó, y cuando se lo conté me dio su tarjeta de visita y me dijo que la llamara si necesitaba cualquier tipo de información. Sentí que de alguna manera había pasado con éxito algún tipo de examen.

Cuando hice una visita a la embajada pasé por delante de ella para ir a hablar con el embajador Lhuillier, hijo de una pareja francesa que se instaló en Cebú, al sur de Filipinas, adoptó la nacionalidad filipina y se hizo rica. En honor del embajador debo decir que reconocía que el color de su piel y su apellido podían explicar la falta de confianza que le tenía la gente. Lo primero que me preguntó al comenzar la entrevista fue qué clase de quejas había oído yo entre la comunidad, y le contesté diciendo que se especulaba con la posibilidad de que el embajador se hubiera enriquecido a costa del dinero de los trabajadores. Parte de su respuesta consistió en hacerme una vista guiada por toda la embajada, a lo largo de la cual señaló una biblioteca recientemente creada, que contaba con una colección de todos los periódicos filipinos y un ordenador a través del cual se podía acceder a la información. Yo le seguí obedientemente a lo largo del tour haciendo una ligera parada sólo cuando me habló de los baños de mármol que había tenido que derribar porque necesitaba más espacio para su despacho. Cuando mis colaboradores que trabajaban para las ONGs se enteraron que había hablado con el embajador me preguntaron qué había comentado acerca de sus protestas. Escucharon las respuestas que me dio y enseguida las desecharon diciendo que las renovaciones eran actos superficiales que no encaraban la verdadera raíz del problema: la necesidad de la migración en Filipinas. Quizá uno de los comentarios que hizo el embajador pueda ser la mejor manera de describir su posición: "Como el resto de las personas que trabajan en la Embajada somos identificados con el propio gobierno filipino e, independientemente de lo que hagamos, siempre van a pensar que nos damos la gran vida a sus expensas".

El hecho de que mis contactos en las ONGs me consideraran una persona útil para conseguir información, y que, al mismo tiempo, el embajador me viese como una herramienta para conseguir saber lo que 
decía la comunidad, me ayudó enormemente. Las noticias que yo conseguía a través de la confianza que inspiraba, variaban enormemente, desde la totalmente mundana hasta la completamente privada, incluyendo desde quién era homosexual hasta quién estaba casado con dos mujeres al mismo tiempo. Quiero decir que se trataba de información que podía haber destruido la reputación de verdaderos pilares de la comunidad, pero el hecho de que yo estaba de paso y no era un miembro de ella, jugó a mi favor porque la gente tenía presente que yo me iba a marchar.

A lo largo de todo este proceso he tenido que enfrentarme al tema de la reciprocidad, el hecho de obtener materiales y experiencias valiosas me ha obligado a plantearme cómo devolver (Narayan 1989: 62), y aparte de pequeños regalos o exquisiteces filipinas, difíciles de encontrar, hechas en Filipinas o en los Estados Unidos, he comprado libros, provisiones, equipamiento y dulces americanos. Sinceramente, me costaba entender por qué los filipinos me pedían siempre chocolate americano, cuando yo misma era una fan del chocolate europeo, sin embargo, con el tiempo he llegado a entender que les recordaba a su casa en Filipinas donde se compraba ese chocolate; se trata, por lo tanto, de un sabor que debe ser entendido, una vez más, como un legado colonial. Siempre hice un esfuerzo para que la colaboración con mis informantes fuese de ida y vuelta, de manera que, además de los pequeños regalos, realicé algunos servicios para la comunidad, tales como actuar de centro de información, lo que implicaba saber cuándo iban a tener lugar las actividades culturales, ayudar a localizar a unos y a otros, proporcionarles información sobre dónde había trabajo, y siempre que salíamos era yo quien pagaba las comidas y las bebidas. Este último aspecto era especialmente importante para mí, no sólo porque era una intelectual tratando de ejercer la reciprocidad, sino como filipina, ya que en mi cultura, como en otras, el intercambio de comida constituye una parte esencial de todos los intercambios sociales (véase Fernandez 1994; Theophano, Curtis y Goode 1984).

En algunas ocasiones, algunos de los encuentros con las mujeres se convirtieron en torrentes de emociones y descripciones sobre la vida en Filipinas y en Italia, en esos momentos yo dejaba de tomar notas y me dedicaba simplemente a escuchar. Ávidas de un oyente empático, llegaban a contarme cosas que ni sus mejores amigas sabían. Al principio yo no sabía qué hacer con este tipo de información, ni siquiera cómo debía ayudar, y además era consciente de que una de las censuras que se le puede hacer a un etnógrafo es la de confundir su vida académica con sus objetivos personales (Vo 2000: 20), pero al final de mi trabajo de campo llegué a comprender que lo único que esperaban de mí era que las escuchara, y que eso era suficiente para ellas. 
La comunidad contaba con figuras de relevancia, además del Padre Bati. Se trataba generalmente de presentadores de radio o líderes de grupos musicales, como por ejemplo Jesse Ramirez, que además de fundador del grupo de cantantes Karilagan, tenía su propio show en la radio y soñaba con ser el primer filipino elegido en Italia para desempeñar un cargo político. Él y las dos docenas que constituyen el grupo musical eran invitados con frecuencia por toda Italia para cantar en actos culturales. En el concierto de diciembre del año 2000 los Karilagan actuaron en una pequeña iglesia próxima a la Fontana de Trevi, la audiencia estaba compuesta por algunos italianos y muchos filipinos. Ofrecieron un repertorio impresionante: canciones americanas, himnos en latín, canciones tradicionales filipinas y canciones italianas. La "Blanca navidad" de Bing Crosby se cantó con un tono nostálgico, a pesar de que el grupo de cantantes, compuesto mayoritariamente por empleados domésticos, nunca había tenido relación ni con la nieve ni con los Estados Unidos (Appadurai 1996). Después de la canción de Bing Crosby, el grupo cantó himnos en latín seguidos de canciones italianas y como final ofrecieron otras tradicionales tagalas de Navidad. En menos de una hora los cantantes presentaron sus historias: su colonialismo español/católico/americano entrelazado con sus experiencias aquí y ahora. Estuve comentando con $\mathrm{Edna}^{4}$, una de las cantantes, la habilidad del grupo para interpretar canciones en distintas lenguas y me contestó:

Si en un mismo concierto cantamos en tagalo, inglés, italiano y latín. ¿ ¿Te imaginas cómo podríamos cantar si no hubiéramos sufrido el colonialismo? Cantamos canciones en tagalo porque es nuestra lengua, en inglés porque tenemos que hacerlo, porque sabemos inglés y porque fuimos colonizados también por los americanos. Cantamos en italiano porque estamos en Italia y sabemos italiano y nos interesa atraer una audiencia italiana, y cantamos, por último, en latín porque es la lengua original de la religión católica que trajeron los españoles.

La competencia cultural de Jesse y su grupo es un motivo de orgullo para la comunidad filipina, puesto que es una forma de demostrar a los italianos que los filipinos "no son sólo trabajadores domésticos", en palabras del propio Jesse. Él mismo se considera un embajador de buena fe que promociona la cultura filipina en Italia y enseña al público sobre ella. Pero al mismo tiempo, los miembros del grupo son también objeto de envidia por parte del resto de la comunidad, algunas personas me han dicho: “Quién se han creído que son para representarnos a todos?". Al

${ }^{4} \mathrm{He}$ cambiado algunos nombres para proteger la identidad de las personas y lo he señalado con un asterisco. 
ejercer este papel y definirse como representantes de la cultura filipina en Italia, el legado cultural del grupo adquiere una mayor legitimidad y, por su parte, Jesse gana la visibilidad y el poder que necesita para acercarse a su sueño de desempeñar un cargo político. Suele ser invitado a las tertulias de la televisión italiana para explicar a la audiencia la cultura de los migrantes filipinos. Yo misma fui entrevistada por Jesse en su propio programa de radio y a partir de mi intervención en él, muchas personas me veían con ojos diferentes; sin embargo, durante el programa pude comprobar que Jesse no pretendía establecer una colaboración igualitaria: era su invitada americana, y aunque yo había pensado que hablaría sobre la situación de los migrantes, Jesse me había llevado en realidad para dar prestigio a su programa como huésped extranjera, ya que de lo que se habló fue, fundamentalmente, de sí mismo.

El grupo de cantantes Karigalan de Santa Pudenziana es uno de los muchos grupos culturales filipinos surgidos en torno a una parroquia particular de Roma, y su importancia para la comunidad, así como para los propios miembros del grupo, no debe subestimarse. Algunas de las funciones que cumple son tan importantes como actuar en lugar de la familia que ha quedado atrás viviendo en Filipinas o de guía en sus vidas sociales y culturales. Me dijeron que el grupo esperaba que todos sus componentes estuvieran presentes durante los ensayos que tienen lugar los jueves por la tarde y los domingos, y aquellos que faltan a una o dos sesiones se considera que dejan de pertenecer a él. También, tiene influencia en la vida amorosa de sus miembros. Esto no es específico sólo de los Kaligalan, otros grupos parroquiales en otras comunidades de Roma prefieren que sus miembros sean "solterones" pues las relaciones amorosas son vistas como algo que puede hacerles abandonar el grupos.

El prestigio de la iglesia católica a los ojos de la comunidad filipina se confirma siempre que un evento cultural cuenta con la presencia de sacerdotes o monjas, y especialmente cuando asiste el Padre Bati. A pesar de los problemas que existen con la embajada filipina, un acontecimiento cultural filipino cobra aún mayor prestigio si asisten a la vez miembros del clero y de la embajada, en caso contrario, mis colaboradoras femeninas consideran menos relevante el evento y suele tener menos audiencia.

Me ha resultado siempre muy difícil determinar la influencia de mis diferentes fuentes de información, clérigos, empleados domésticos, miembros de la embajada, trabajadoras feministas de las ONGs, sobre la propia información que me facilitaban, de hecho ha supuesto un verdadero

${ }^{5}$ Datos obtenidos a través del trabajo de campo. 
desafío epistemológico. Este problema es comparable a los sesgos que un antropólogo o un folklorista introduce en su propia etnografía (Clifford y Marcus 1986), pero en mi propio caso soy consciente además de que las experiencias vitales y las creencias de mis colaboradores influyen en la producción de su conocimiento. Un ejemplo del conflicto planteado entre el conocimiento y las políticas del espacio lo constituye la siguiente descripción de una Fiesta de Navidad, celebrada en el Colegio Filipino (un seminario para sacerdotes filipinos en Roma) que tuvo lugar una noche de diciembre del año 2000.

La fiesta consistió en una misa y a continuación los sacerdotes conmemoraron la estación de Adviento con una pequeña actuación integrada por canciones católicas e himnos cantados en latín, tagalo e inglés. Después se sirvió una comida a base de arroz hervido con jengibre y pollo, que es un plato que suelen tomar en Filipinas los enfermos y los bebés, pero que disfruta todo el mundo. A continuación se ofreció un postre dulce llamado ginatan que se elabora con harina de arroz. A pesar de que el lugar está emplazado lejos del centro de Roma (de hecho se encuentra en un barrio fuera de la ciudad, a unos 40 minutos en metro del centro, seguidos de un paseo de 15 minutos por una transitada autopista), la asistencia a la fiesta fue masiva. El local constaba de una habitación sin asientos en la que actuaban los sacerdotes y una antesala llena de grupos de filipinos hablando. La cola del cuarto de baño ocupaba unos diez metros y llegaba hasta la antesala.

Yo tuve noticia de este evento unos días antes, gracias a unos filipinos que encontré en la calle. Todos ellos se refirieron al acontecimiento como una ocasión para divertirse y comer gratis, pero nadie mencionó ni la misa ni la actuación de los sacerdotes. Tampoco supo nadie explicarme su significado, que no era otro, obviamente, que celebrar la Navidad.

Por el contrario, para los sacerdotes que entrevisté en el Colegio, se trataba de una ocasión cuyo objetivo consistía en traer a Italia tradiciones culturales filipinas y, al mismo tiempo, conseguir que la gente cumpliera sus obligaciones religiosas de una forma estructurada. En opinión del Padre Mario Sanchez, que está haciendo una tesis doctoral sobre la espiritualidad, el Colegio solía ser el lugar de reunión más importante de los migrantes en Roma, antes de que Santa Pudenziana y la capellanía fuera instituida para atender las necesidades de estas personas. Los sacerdotes, sin embargo, se encontraban saturados y eran incapaces de atender todas las demandas sociales y religiosas de los migrantes, de manera que sus recursos y su tiempo eran acaparados por la comunidad en detrimento de sus estudios. De forma que actualmente el Colegio organiza al menos dos eventos culturales, uno en Navidades y otro en mayo, pero su obje- 
tivo principal es el de ser una institución educativa, lo que, en opinión del Padre Sanchez, no entendía la comunidad: "Nuestro principal propósito aquí no es ése (organizar eventos para los migrantes), sino los estudios", me dijo Sanchez, "eso tiene que quedar muy claro y es la razón por la cual ni siquiera la comunidad puede pedir sacerdotes aquí... porque hemos sido enviados a Roma para estudiar".

Ya que tanto los migrantes como los sacerdotes constituyeron para mí fuentes de conocimiento a lo largo de mi trabajo, tuve que entender cómo ese conocimiento suyo se legitimaba y cómo se teñía a partir de las diferencias de percepción y formas de conocer. Ambas fuentes, migrantes y sacerdotes, compartían conocimientos que hacían legítimos a través de sus experiencias. Los migrantes creían que la fiesta de Navidad del Colegio era un sitio donde conseguir comida y diversión, mientras que los sacerdotes la consideraban como una forma de cumplir las obligaciones cristianas y una manera de lograr que los migrantes se acercaran a la iglesia y a su cultura. Los sacerdotes de Colegio pensaban además que la fiesta de Adviento era una forma de prevenir una mayor intrusión de su espacio y de su tiempo. Se dijo que, a pesar de las diferencias en las formas de entenderla, la fiesta del año 2000 fue un éxito completo. Las disparidades en la producción del conocimiento pueden ser también un factor que juega en las políticas del espacio, como voy a ilustrar a continuación.

La tarde del 27 de mayo del año 2001 asistí a una "fiesta de verano" auspiciada por la comunidad de la iglesia de San Leone y organizada por el Padre Atilano Fajardo (conocido también como Padre Nonong). Esta comunidad está constituida por unos 27 miembros de los cuales la mayoría procedía de la provincia de Batangas en Filipinas. El Padre Atilano me informó que iba a haber comida y juegos, tanto americanos como filipinos. Llegué a la iglesia con una amiga, Selica*, después de un polvoriento viaje en tranvía. No había señales de filipinos por ninguna parte, aunque en el fresco interior de mármol de la iglesia un grupo de italianos practicaban sus canciones. Salí y empecé a preguntar por el paradero del grupo filipino, y entonces lo escuché; se trataba de la voz de un hombre filipino hablando en tagalo a través de un altavoz, y cuando la brisa empezó a jugar con nuestro pelo, unos momentos después, distinguí el sabroso olor característico de la barbacoa filipina, marinada en vinagre y en ajo. Seguimos los sonidos y los olores a través de una caótica calle desde la iglesia hacia una zona llena de edificios y, después de doblar una esquina y subir una pequeña colina, llegamos ante un grupo de adolescentes filipinos que jugaban al voleibol en un pequeño parque. El lugar no se podía ver desde la iglesia y me sorprendió pensar que si 
cerraba los ojos y usaba sólo mis oídos y mi nariz, me podía trasladar con toda facilidad a cualquier pueblo del centro de Filipinas o a una fiesta en el parque en Carson, California, donde viven muchos filipinos. Utilizando únicamente mis sentidos no-visuales ganaba sabiduría o conocimiento sobre el lugar de mi trabajo de campo (Howes 1991: 4), gracias a que utilicé mis otros sentidos, y no el de la vista, fui capaz de entender la "poética cultural" (Clifford y Marcus 1986: 12) en la que distintos datos sensoriales, voces, olores, acentos, y no sólo imágenes, me condujeron al lugar donde las actividades culturales estaban teniendo lugar.

Escribí en mi diario de campo la siguiente descripción del evento:

El lugar de encuentro era una pradera de césped verde rodeada de grandes edificios de viviendas. En uno de los laterales quedaban los restos de un muro antiguo que proyectaba amplificado el ruido a todas las viviendas del entorno.

La falta de espacio era evidente, lo mismo que el choque de culturas. El parque era muy pequeño y estaba protegido en uno de sus lados por el muro en ruinas o una fortaleza. La fiesta estaba en marcha completamente cuando yo llegué. Resultó difícil encontrarla, pero seguí simplemente el olor de la barbacoa. Los niños jugaban a la pelota, el altavoz atronabá música junto con las voces de los presentadores. Yo me preguntaba si la gente se quejaría.

Y desde luego se quejó. Una hora más tarde (a las 18 horas), y después de una intensa mirada de una pareja italiana que pasaba con su perro, un italiano de edad vino a preguntar si el Padre Nonong era el responsable; cuando Nonong dijo que sí, el hombre comenzó su bombardeo de quejas por el ruido, decía que los sonidos hacían eco y que debían limpiar el parque. Nongong accedió a todas sus demandas, pero el hombre siguió protestando. En un momento determinado el italiano dijo que a él le gustaba la cultura de los filipinos (lo que ya había dicho al principio de su discurso), y que era estupendo que los filipinos hiciesen celebraciones, pero que el parque era un lugar compartido por todos y que los filipinos debían ser considerados con los demás. Le oí decir: Sono un amico y después continuó en italiano "solamente quería decirle cómo me sentía", y continuaba diciendo: "soy un amigo, me gusta este lugar, pero...". Dijo además que por la noche era un sitio usado por los drogadictos (de hecho mencionó los migrantes "negros") y que por eso era fácil que los niños acabaran clavándose agujas infectadas. Así es el espacio en Roma, se pelea mucho. Por este pequeño recinto compiten, y chocan, tres grupos: italianos, nigerianos y filipinos.

El comentario de este hombre que más me llamó la atención fue cuando describió la reunión de filipinos como " $E$ 'un casino" (es un desorden, un caos). Cuando los adolescentes filipinos estaban jugando al voleibol, chillando y riendo con verdadero placer, les señalaba diciendo: "¿Ve lo que quiero decir? El ruido que hacen los niños es realmente estridente". Parece que en Roma uno no sólamente debe negociar el espacio y el tiempo, sino además el derecho de reír a carcajadas en público.

Mi experiencia durante el trabajo de campo revela la importancia que tiene la religión para el filipino medio migrante en Italia y, al igual que 
ocurre en otros lugares y en otros momentos, la religión significa más para los filipinos que se encuentran trabajando lejos de casa, en la diáspora (véase Warner y Wittner 1998: 3; Pozzetta 1991). Los filipinos son famosos por su fe religiosa, quizá más que otros migrantes en Italia. Más del $80 \%$ de la población en Filipinas es católica, el hecho de que Roma sea la capital de la fe católica atrae a muchos (Cuchapin 2000: 220). Un ejemplo puede verse en San Leone donde, debido a la falta de locales y espacios para reunirse, y también a la carencia de servicios sociales, los filipinos en Italia miran hacia la iglesia para algo más que satisfacer sus necesidades religiosas. Los sacerdotes como el Padre Nonong lo prefieren así porque, en sus propias palabras, "si no están en la iglesia, están jugando".

La iglesia católica de Roma ofrece a los migrantes espacio para celebrar sus actividades culturales que tengan una dimensión religiosa. En contraste, las actividades puramente seculares como un juego popular filipino llamado tong-it (parecido al poker) y otros tipos de celebraciones no religiosas, tienen lugar en pisos privados o en piazzas. Como el juego es una actividad prohibida, los filipinos que juegan a las cartas en las piazzas, siempre están vigilando que no venga la policia. Todo tipo de intercambio de dinero se hace de manera encubierta, las actividades "ilícitas" están caracterizadas por su puesta en escena cerrada, los acontecimientos "legítimos", tanto para la comunidad filipina como para la sociedad italiana, tienen lugar en los espacios abiertos de las iglesias. En Santa Pudenziana, que aloja el centro de los migrantes filipinos, los actos se celebran en los espacios alrededor de la iglesia o dentro del vestíbulo, la comida se dispone en una de las paredes laterales del vestíbulo y los jóvenes juegan al voleibol en un campo cubierto. En sus días libres, los filipinos se suelen reunir en las piazzas y en las estaciones de autobús y en la estación Termini, pero muchos prefieren acudir al centro de la iglesia local. De las 900 iglesias católicas que hay en Roma, los filipinos han formado pequeñas comunidades en unas 50; ello significa que van a las iglesias a reunirse con sus amigos, a organizar actividades culturales y a comer comida típica filipina. En San Silvestro, por ejemplo, todos los jueves por la tarde y los domingos el espacio encima de la iglesia se llena de vendedores que ofrecen productos comestibles típicamente filipinos, tales como lumpia, rollos de huevo, fideos pancit y otros aperitivos y bebidas nativos.

El papel que desempeña la iglesia católica en las vidas de las mujeres filipinas no debe subestimarse tampoco. Muchas de las que trabajan como empleadas del hogar tratan de soportar, y de hecho soportan, la dureza de su trabajo porque se consideran "los nuevos apóstoles 
de Cristo"6. Los sacerdotes como el Padre Bati consideran a los filipinos en Italia como evangelizadores de Jesús, y esto es así porque las filipinas tienen acceso a la esfera privada de las familias italianas, tienen influencia sobre los niños y, consecuentemente, sobre las mismas personas que les emplean. Algunas mujeres me han dicho que a veces llevan a los niños a la iglesia; sus padres también van, e incluso cuando éstos no van, confían sus hijos a las filipinas, de manera que la religión ayuda a aliviar la tensión entre empleadas y empleadores.

En cuanto al futuro, algunos filipinos optan por retornar a Filipinas y que sus hijos permanezcan allí, sin embargo, la mayoría prefiere quedarse en Italia y aprovechar las ventajas de las leyes de reunificación familiar. Por este motivo se ha incrementado el número de niños y adolescentes, algunos han nacido en Italia, pero la mayoría son reclamados a Filipinas por madres que no les han visto crecer. Muchos adolescentes llegaron el Día mundial de los jóvenes que tuvo lugar en agosto del año 2000 y no volvieron. Algunos van al colegio y otros encuentran trabajo en las casas, como sus padres. ...

¿Qué va a ocurrir cuando la comunidad crezca y madure? Las migrantes filipinas, al igual que otras mujeres migrantes, deben ser reconocidas como las que "llevan el dinero a casa". Sus necesidades deberían tenerse en cuenta, así como el hecho de que los únicos lugares a los que pueden acudir para ello, hoy por hoy, es a sus propias redes de relaciones y a las organizaciones eclesiales, pero no a los servicios sociales del gobierno italiano. Deben instituir más sitios a los que puedan acudir a buscar ayuda. Además debería prestarse atención a la migración de reemplazo, ya que cuando las madres dejan el trabajo doméstico son sustituidas por sus hijas. El nuevo flujo de migración significa que las mujeres más jóvenes se dirigen en masa hacia Italia y que retrasan la constitución de sus propias familias. ¿Cómo afectará este hecho a la sociedad filipina? No sólo los temas de género, sino también los de etnicidad y clase deberán ser tenidos en cuenta en futuros estudios sobre migración, y sólo el tiempo decidirá si la segunda y tercera generación de filipinos en Roma van a constituir una clase perpetua de trabajadores domésticos. Personalmente, tengo la esperanza de que no sea así.

\section{BIBLIOGRAFÍA CITADA}

Alabastro, RubÉn P. 1995. "Philippine exodus leads to death row". The Washington Times. Friday, September 22.

\footnotetext{
${ }^{6}$ Entrevista con Rhacel Parrenas en Filadelfia, Pennsylvania, abril, 1999.
} 
ALEGADO, DEAN. 1992. The Political Economy of International Labor Migration from the Philippines. Tesis doctoral. Hawai: Dept. of Political Sciences, University of Hawai. ANDAll, JaCQueline. 1998. "Catholic and State Constructions of Domestic Workers. The Case of Cape Verdean Women in Rome in the 1970s.", en K. Koser y H. Lutz (eds.), The New Migration in Europe. Social Constructions and Social Realities. Londres: Macmillan Press.

ANDERSON, BENEDICT. 1998. The Spectre of Comparisons. Nacionalism, Southeast Asia and the World. Londres y Nueva York: Verso.

ANTHIAS, FlOYA. 2000. "Metaphors of Home: Gendering New Migrations to Southern Europe", en F. Anthias y G. Lazaridis (eds.), Gender and Migration in Southern Europe. Women on the Move: 15-47. Nueva York: Berg.

- y Gabriella Lazaridis (eds.). 2000. Gender and Migration in Southern Europe. Women on the Move. Nueva York: Berg.

APPADURAI, ARJun. 1996. Modernity at Large. Minneapolis: University of Minnesota Press.

Briggs, Charles L. 1986. Learning How to Ask. A Sociolinguistic Appraisal of the Rol of the Interview in Social Sciences Research. Cambridge: Cambridge University Press.

CAMPANI, GIOVANNA. 2000. "Immigrant Women in Southner Europe: Social Exclusion, Domestic Work and Prostitution in Italy", en K Rusell, G. Lazaridis y Ch. Tsardanidis (eds.), Eldorado or Fortress? Migration in Southern Europe: 147-169. Nueva York: St. Martin's Press.

Carino, Benjamin V. (ed.). 1998. Filipino Workers on the Move: Trends, Dilemmas and Policy Options. Manila: Philippine Migration Research Network.

CARITAS DI ROMA. 2000. Immigrazione. Dossier Statistico 2000.

CATHOLIC INSTITUTE FOR INTERNATIONAL RELATIONS. 1987. The Labour Trade: Filipino Migrant Workers around the World. Londres: CIIR.

CHELL, VICTORIA. 1997. "Gender-Selective Migration: Somalian and Filipina Women in Rome", en K. Russell y R. Black (eds.), Southern Europe and the New Immigrations: 75-92. Sussex: Academic Press.

Chell-RoBinson, VictoRiA. 2000. "Female Migrants in Italy: Coping in a Country of New Immigration", en F. Anthias y G. Lazaridis (eds.), Gender and Migration in Southern Europe. Women on the Move. Nueva York: Berg.

Clifford, JAmes y George E. MARCus. 1986. Writing Culture: The Poetics and Politics of Ethnography. Berkeley: University of California Press.

COHEN, RoBin y STEVEN VerTOVEC (eds.). 1999. Migration, Diasporas, and Transnacionalism. Cheltenham-Northampton: Edward Elgar.

COLE, JefrReY. 1997. The New Racism in Europe: A Sicilian Ethnography. Cambridge: Cambridge University Press.

CONSTABLE, NicOlE. 1997. Maid to Order in Hong Kong. Stories of Filipina Workers. Ithaca y Londres: Cornell University Press.

CuCHAPIN, PAGaSA. 2000. I Nuovi "Eroi": Etnografia dell'immigrazione Filippina in Italia. Tesis doctoral, Universitá degli Studi di Roma, La Sapienza.

Eviota, Elizabeth Uy. 1992. The Political Economy of Gender. Women and the Sexual Division of Labour in the Philippines. Londres: Zed Books.

FERNÁNDEZ, DOREEN G. 1994. Tikim: essays on Philippine food and culture. Pasig, Manila: Anvil Publishing.

Go, STella P. 1998. "Towards the $21^{\text {st }}$ Century: Whither Philippine Labor Migration?", en B. V. Carino (ed.), Filipino Workers on the Move: Trends, Dilemmas and Policy Options: 9-44. Manila: Philippine Migration Research Network. 
Howes, DAvid. 1991. The Varieties of Sensory Experience. Toronto: University of Toronto Press.

Ileto, Reynaldo. 1979. Pasyon and Revolution. Popular Movements in the Filippines, 1840-1910. Quenzon City: Ateneo de Manila University Press.

IOSIFIDES, THEODOROS. 1997. "Immigrants in the Athens Labour Market: a Comparative Study of Albanians, Egyptians and Filipinos", en R. King y R. Black (eds.), Southern Europe and the New Immigrations: 26-50. Sussex: Academic Press.

Kang, Miliann. 2000. "Researching One's Own: Negotiating Co-Ethnicity in the Field", en M. Manalansan (ed.), Cultural Compass: Ethnographic Explorations of Asian America: 38-48. Filadelfia: Temple University Press.

KING, Russell. 2000. "Southern Europe in the Changing Global Map of Migration", en King, Lazaridis y Tsardanidis (eds.), Eldorado or Fortress? Migration in Southern Europe: 3-26. NuevaYork: St. Martin's Press.

King, Russell; Gabriella lazaridis y Charalambos TSARDanidis (eds.). 2000. Eldorado or Fortress? Migration in Southern Europe. Nueva York: St. Martin's Press.

Koser, Khalid y Helma LuTz (eds.). 1998. The New Migration in Europe. Social Constructions and Social Realities. Londres: Macmillan Press.

LAZARIDIS, GABRIELlA. 2000. "Filipino and Albanian Women Migrant Workers in Greece: Multiple Layers of Oppression", en F. Anthias y G. Lazaridis (eds.), Gender and Migration in Southern Europe. Women on the Move: 49-79. Nueva York: Berg.

NARAYAN, KIRIN. 1989. Storytellers, Saints, and Scoundrels. Folk Narrative in Hindu Religious Teaching. Filadelfia: University of Pennsylvania Press.

PARRENAS, RHACEL. 1998. The Global Servants: Immigrant Filipina Domestic Workers in Rome and Los Angeles. Tesis doctoral. Dept. de Sociología. University of California. Berkeley.

PhizaCKLEA, ANNIE. 1998. "Migration and Globalization: A Feminist Perspective", en K. Koser y H. Lutz (eds.), The New Migration in Europe. Social Constructions and Social Realities: 21-38. Londres: Macmillan Press.

POZZETTA, GEORGE E. (ed.). 1991. The Immigrant Religious Experience. Nueva YorkLondres: Garland Publishing.

RAFAEL, VICENTE. 2000. White Love and otber events in Filipino History. Durham y Londres: Duke University Press.

Theophano, JANET; KAREN CurTis y Judith GoOdE. 1984. "A Framework for the Analysis of Continuity and Change in Shared Socio-cultural Rules for Food Use: the ItalianAmerican Pattern", en Brown y Mussell (eds.), Ethnic and Regional Foodways in the United States. The Performance of Group Identity: 66-90. Knoxville: The University of Tennessee Press.

VANZI, SOL. 1998. "\$7 Overseas Filipino Workers' Earning (OFW) Buoy Economy in '98" (artículo obtenido en internet el 15 de enero de 1998, de la revista Philippine Headline News Online (http://www.newsflash.org/199812/hlframe.html).

VO, LINDA TRINH. 2000. "Performing Ethnography in Asian American Communities: Beyond the Insider-versus-Outsider Perspective", M. Manalansan (ed.), Cultural Compass: Ethnographic Explorations of Asian America: 17-37. Filadelfia: Temple University Press.

WARNER, R. STEPHEN y JUDITH G. WITTNER. 1998. Gatherings in Diaspora. Religious Communities and the New Immigration. Filadelfia: Temple University Press. 